\title{
Two different hematocrit detection methods: Different methods, different results?
}

\author{
Marco Bosshart ${ }^{+1}$, John F Stover ${ }^{+1}$, Reto Stocker ${ }^{1}$, Lars M Asmis², Jörg Feige1, Thomas A Neff', Reto A Schuepbach1, \\ Silvia R Cottini ${ }^{1}$ and Markus Béchir*1
}

\begin{abstract}
Background: Less is known about the influence of hematocrit detection methodology on transfusion triggers. Therefore, the aim of the present study was to compare two different hematocrit-assessing methods. In a total of 50 critically ill patients hematocrit was analyzed using (1) blood gas analyzer (ABLflex 800) and (2) the central laboratory method (ADVIA 2120 ) and compared.

Findings: Bland-Altman analysis for repeated measurements showed a good correlation with a bias of $+1.39 \%$ and 2 SD of $\pm 3.12 \%$. The $24 \%$-hematocrit-group showed a correlation of $r^{2}=0.87$. With a kappa of $0.56,22.7 \%$ of the cases would have been transfused differently. In the-28\%-hematocrit group with a similar correlation $\left(r^{2}=0.8\right)$ and a kappa of $0.58,21 \%$ of the cases would have been transfused differently.

Conclusions: Despite a good agreement between the two methods used to determine hematocrit in clinical routine, the calculated difference of $1.4 \%$ might substantially influence transfusion triggers depending on the employed method.
\end{abstract}

\section{Findings}

Measurement of hemoglobin or hematocrit is of major importance in contemporary patient care. Anemia or traumatic/surgical blood loss might require transfusion of red blood cells (RBC) to increase oxygen delivery and counteract tissue hypoxia. However, clear transfusion triggers are difficult to define because there is no clear delineation between risk and benefit. To date, at least 3 large randomized controlled trials have compared restrictive transfusion triggers to a more liberal transfusion regime in critically ill adult patients[1], pediatric intensive care patients[2] and premature infants [3]. All three studies showed that a restrictive transfusion regime reduced RBC transfusion requirements without increasing morbidity and mortality. In this context, hemoglobin concentrations ranging from $7-9 \mathrm{~g} / \mathrm{dl}$ were compared to $10-12 \mathrm{~g} / \mathrm{dl}$.

In this context, we have observed a discrepancy between the hematocrit values determined by routine arterial

\footnotetext{
*Correspondence: markus.bechir@usz.ch

1 Surgical Intensive Care Medicine, University Hospital of Zurich, CH 8091

Zurich, Switzerland

+ Contributed equally

Full list of author information is available at the end of the article
}

blood gas analysis compared to routine analysis in our central laboratory. Therefore, we analyzed 250 blood samples taken from 50 critically ill patients. The aims were (1) to compare two different hematocrit-testing methods routinely used in our intensive care unit (ICU) and hospital (central laboratory) and (2) to determine whether these two methods might provide different transfusion triggers.

In accordance with the principles outlined by the World Medical Association declaration of Helsinki and following approval by the local Ethics Committee which waived the need for written informed consent for this post hoc data analysis, patient data from 50 patients treated on our intensive care unit from November 2007 to March 2008 were analyzed retrospectively. A total of 50 critically ill patients treated consecutively in our department were included irrespective of hemodynamic stability or diagnosis for the comparison of the two methods.

Blood was drawn daily, usually, at 6 a.m. for routine laboratory analysis, including 2 hematograms performed with 2 different methods. To compare hematocrit values determined by these two different methods of analysis, 250 paired hematocrit values (data of 50 patients over 5 
consecutive days resulting in 250 paired samples) were considered. The patients were assigned to 2 different groups depending on the transfusion targets set during ICU treatment. A total of 20 patients with severe traumatic brain injury, severe burns, following reconstructive surgery or with cardiac ischemia had a hematocrit target of $28 \%$. The other 30 patients had a target hematocrit of $24 \%$.

On the ICU arterial blood samples were routinely drawn in 4 to 6 hour intervals to control $\mathrm{paO}_{2}, \mathrm{paCO}_{2}$, hematocrit, glucose or potassium serum levels, required to optimize ventilatory settings, guide transfusions, and adapt intravenous infusion of insulin or potassium. These blood samples were analysed by the nurses using the commercially available blood gas analyzer ABLflex 800 (Radiometer Medical, Copenhagen, Denmark; http:// www.Radiometer.com) located on our ICU. Blood was drawn daily, usually, at 6 a.m. for routine laboratory analysis. Among others, differential blood count including analysis of hemoglobin/hematocrit was performed. As a standardized pre- analytic procedure[4,5], at least $2 \mathrm{ml}$ of blood were discarded to prevent hemodilution by infused fluids before withdrawing blood for the actual analysis using specialized syringes, i.e., the syringe for the ABLflex 800 (Arterial Blood sampler $1.7 \mathrm{ml}$, Radiometer Copenhagen, Denmark) and vacutainers (BD Vacutainer K2E $5.4 \mathrm{mg}$, BD-Plymouth, PL67BP, UK) for the ADVIA 2120.

\section{Method 1: ABLflex 800}

The specimen is drawn from the syringe into the cuvette within the gas analyzer maintained at $37^{\circ} \mathrm{C}$. Thereafter, one microliter of the specimen is hemolized via ultrasound $(30 \mathrm{kHz})$. Hemoglobin content is assessed spectrophotometrically using 128 different wave lengths (478 to $672 \mathrm{~nm}$ ). The light is transmitted via glass-fiber optics through a diffraction gating, which diffracts the light into 128 single wavelengths. The detecting device consists of 128 photo diodes. According to the equation of LambertBeer hemoglobin content (ctHb) of the blood sample is determined. Based on the ctHb the hematocrit (hct) is calculated via an internal algorithm.

\section{Method 2: ADVIA 2120}

(Siemens Medical Solutions Diagnostics, Zurich, Switzerland, http://www.diagnostics.siemens.com): This device uses 2 sequential methods of haemoglobin measurement:

(1) Flow cytometry: In a first step red blood cells are applied to iso-volumetric sphering and partial fixation. Then a second reagent encases the sample stream and the entire specimen passes through a flow cell. A red laser then measured cell volume and intracellular haemoglobin concentration. The amount of light scattered at low angle $\left(2-3^{\circ}\right)$ is dependent on the cell volume and the high angle $\left(5-15^{\circ}\right)$ is related to the refractive index of the cell, reflecting the haemoglobin concentration for red blood cells (CHCM). The haematocrit then is calculated.

(2) A cyanide-free reagent (borate solution) and a surfactant $(\mathrm{N}, \mathrm{N}$,-dimethyllaurylamine $\mathrm{N}$-oxide) cause haemolysis and facilitate the oxidation of haeme iron to $\mathrm{Fe} 3^{+}$. Because of the alkaline $\mathrm{pH}$ of the reagent haemoglobin loses most of its salt bridges. The ligated heme groups are solubilized by surfactant micelles to generate a green end-product which can be detected photometrically[6].

During analysis both values for haemoglobin determined by 1 and 2 are compared by an internal algorithm. In case of a predefined discrepancy the haemoglobin value is corrected in respect to the colorimetric method which is considered as the "gold standard" [7].

Our null hypothesis was that the two different methods (ABLflex 800 and ADVIA 2120) were equal and did not provide different transfusion triggers. The alternative hypothesis was that the ABLflex 800 method is not equal to the ADVIA 2120 method.

Bland-Altman analysis corrected for repeated measurements [8,9] and Cohen's kappa statistics [10] were used to compare the two methods (Statview 4.5, abacus concepts, Berkeley, CA, USA). Cohen's kappa statistics compares the observations of two different methods and therefore results in 4 possible cases: $+/+,+/-,-/+$ and $-/-$. There is an agreement of the methods in the cases of $+/+$ and $-/$ (i.e. "transfused"/"transfused" and "not transfused"/"not transfused") and there is no agreement in the cases +/and -/+ (i.e.,"transfused"/"not transfused" and "not transfused"/"transfused"), respectively. With kappa $\leq 0.4$ the agreement is poor, if kappa is between 0.4 and 0.75 agreement is fair and kappa $\geq 0.75$ reflects excellent agreement between these two methods.

Comparisons between groups were calculated either with Chi-square test, with a nonparametric Mann-Whitney test or ANOVA log rank test (Statview 4.5, abacus concepts, Berkeley, CA, USA). Statistical significance level was accepted with $\mathrm{p}<0.05$.

The investigated 50 patients were grouped according to the pre- defined transfusion triggers (24 and $28 \%$ ) used on our intensive care unit for different illnesses, resulting in 30 patients within the 24-\%-hematocrit-group and 20 patients in the $28-\%$-hematocrit-group. As depicted in table 1 these two hematocrit target groups consist of heterogeneous patients with different leading diagnosis which, in turn, dictated different treatment concepts and different transfusion triggers. Consequently, these 2 groups are not comparable. Serum levels of lactate and body temperature were significantly different in the 2 groups ( $\mathrm{p}=0.02$ and 0.04 , respectively). Hospital mortality was also significantly different $(\mathrm{p}=0.03)$. 
Table 1: Baseline and clinical characteristics

\begin{tabular}{|c|c|c|c|}
\hline Parameter & 24\%-hct-group & 28\%-hct-group & p-value \\
\hline Number of patients & 30 & 20 & \\
\hline Men & $21(70 \%)$ & $15(75 \%)$ & 0.70 \\
\hline Women & $9(30 \%)$ & $5(25 \%)$ & 0.70 \\
\hline Age (yrs.) & $52.5 \pm 13.6$ & $44.8 \pm 28$ & 0.17 \\
\hline Heart rate (bpm) & $94 \pm 24$ & $83 \pm 22$ & 0.07 \\
\hline $\mathrm{MAP}(\mathrm{mmHg})$ & $76 \pm 15$ & $83 \pm 11$ & 0.11 \\
\hline Weight (kg) & $87.2 \pm 34.7$ & $70.8 \pm 11.4$ & 0.10 \\
\hline Height (cm) & $172 \pm 10$ & $174 \pm 10$ & 0.70 \\
\hline \multicolumn{4}{|l|}{ Diagnosis } \\
\hline Sepsis & 11 (36.7\%) & 0 & \\
\hline Lung-TPL & $3(10 \%)$ & 0 & \\
\hline Liver-TPL & $2(6.7 \%)$ & 0 & \\
\hline Fascitis & $1(3.3 \%)$ & 0 & \\
\hline Mesothelioma & $1(3.3 \%)$ & 0 & \\
\hline Liver Cirrosis & 1 (3.3\%) & 0 & \\
\hline Colon-Carcinoma & $1(3.3 \%)$ & 0 & \\
\hline Severe Brain injury & 0 & $9(45 \%)$ & \\
\hline Polytrauma & $8(26.7 \%)$ & $2(10 \%)$ & \\
\hline Severe burn injury & 0 & $8(40 \%)$ & \\
\hline ARDS & $1(3.3 \%)$ & 0 & \\
\hline Gastric bypass & $1(3.3 \%)$ & 0 & \\
\hline Cardiac arrest & 0 & $1(5 \%)$ & \\
\hline Lactat $(\mathrm{mmol} / \mathrm{l})$ & $1.7 \pm 1.8$ & $1.2 \pm 0.7$ & 0.02 \\
\hline Temp. $\left({ }^{\circ} \mathrm{C}\right)$ & $37.0 \pm 1.5$ & $36.7 \pm 1.3$ & 0.04 \\
\hline $\begin{array}{l}\text { Blood glucose } \\
(\mathrm{mmol} / \mathrm{l})\end{array}$ & $6.7 \pm 1.3$ & $6.7 \pm 1.9$ & 0.15 \\
\hline $\begin{array}{l}\text { Norepinephrine } \\
\text { (microg/min) }\end{array}$ & $7.9 \pm 11.5$ & $8.3 \pm 12.8$ & 0.10 \\
\hline SAPS II & $39 \pm 17$ & $31 \pm 15$ & 0.07 \\
\hline Hospital mortality & $15(50 \%)$ & $4(20 \%)$ & 0.03 \\
\hline
\end{tabular}

Bland-Altman analysis for repeated measurements of all patients showed a good agreement with a bias of $+1.39 \%$ and 2 standard deviations (2 SD) of $\pm 3.12 \%$ (figure 1 ). Thus, the ABL800 flex method showed an approximately $1.4 \%$ higher hematocrit than the ADVIA 2120 method. Bias for each group was not statistically different (1.11 \pm
$1.83 \%$ in the $24 \%$-hematocrit-group vs. $1.70 \pm 1.24 \%$ in the 28\%-hematocrit-group, $\mathrm{p}=0.26$ ).

According to the targeted transfusion triggers of $24 \%$ and $28 \%$ two kappa statistical analysis were performed in 30 patients with a transfusion trigger set at hematocrit of $24 \%$ and 20 patients with a hematocrit transfusion trigger 


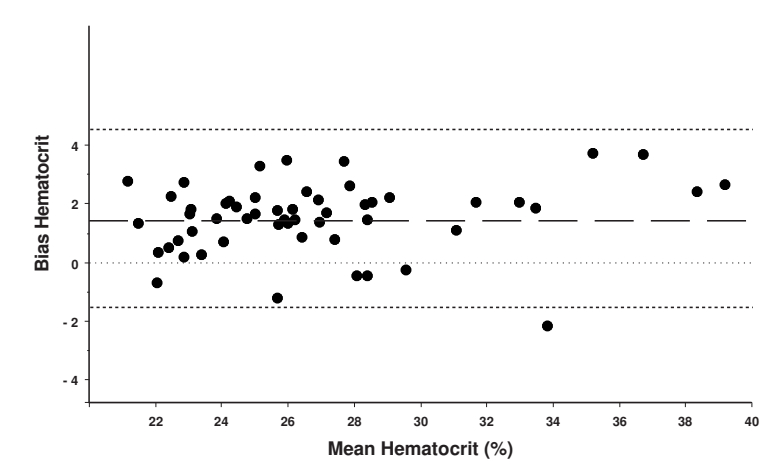

Figure 1 Bland-Altman analysis for repeated measurements of all patients showed a good agreement with a bias of $+1.39 \%$ and 2 standard deviations ( $2 \mathrm{SD}$ ) of $\pm \mathbf{3 . 1 2} \%$. The lower and upper limits of agreement (bias \pm 2 SD) are -1.73 and $4.51 \%$, respectively. Thus, the ABLflex 800 method showed elevated hematocrit by approximately $1.4 \%$.

of $28 \%$. In those patients with a predefined hematocrit target of $24 \%$ ( $n=150$ values) both methods showed a significant correlation of $\mathrm{r}^{2}=0.87$ (figure 2). Kappa of 0.56 indicated a fairly good agreement of the methods, i.e., values located within the lower field below $24 \%$ (square III) reflect patients who needed to be transfused according to both methods of analysis and square I depicts those patients in whom transfusions were not

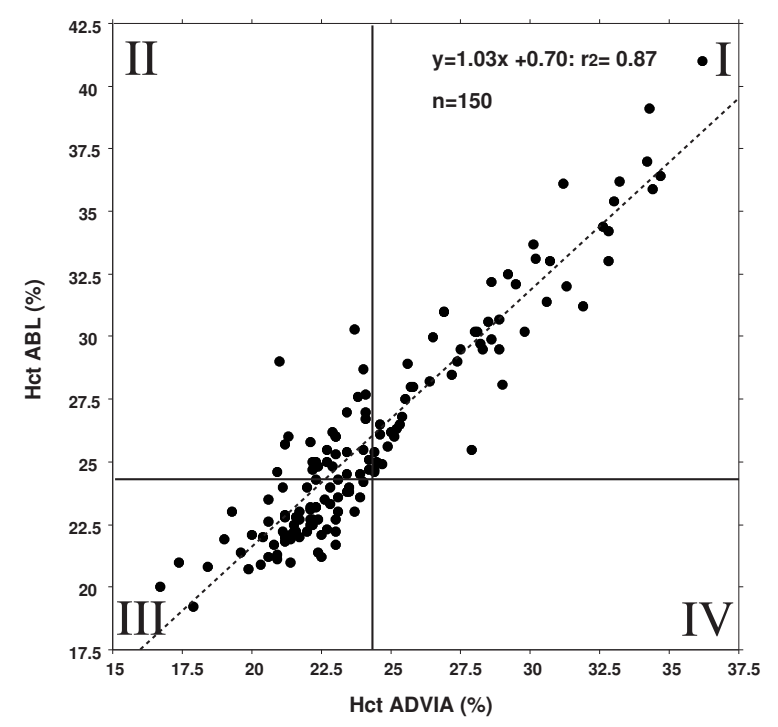

Figure 2 In 30 patients with a predefined hematocrit target of $24 \%$ ( $n=150$ values) both methods showed a significant correlation of $r^{2}=0.87$ and kappa was 0.56 , indicating a fair agreement of the methods. Values located within the lower field below $24 \%$ (square III) reflect patients who need to be transfused according to both methods of analysis; in contrast, square II shows 34 patients (22.7\%) who would have been transfused according to the hematocrit values determined by the central laboratory. required, regardless of analytical procedure. In contrast, square II shows 34 patients (22.7\%) who would have been transfused according to the hematocrit values determined by the central laboratory. According to the blood gas analysis, however, these patients would not have been transfused.

In patients with predefined hematocrit target of $28 \%(\mathrm{n}=$ 100 values) both methods also correlated well with $\mathrm{r}^{2}=$ 0.8 (figure 3 ). Kappa of 0.58 also reflected a fairly good agreement of these two methods, i.e., values located within square III below $28 \%$ reflected patients requiring RBC transfusions according to both methods of analysis. Square II shows 21 patients (21\%) in whom hematocrit determined by the central laboratory would have suggested RBC transfusion while blood gas analysis would not have indicated RBC transfusion. In square I none of the patients would be transfused, regardless of analytical procedure.

According to the present analysis, ADVIA 2120 and ABLflex 800 provide similar hematocrit values. Nevertheless, values close to the lower transfusion/hematocrit threshold will be strongly influenced by the employed analytical method. Thus, these methodological differences must be considered in addition to the defined transfusion trigger to standardize transfusion practice within the individual ICU/hospital and across different

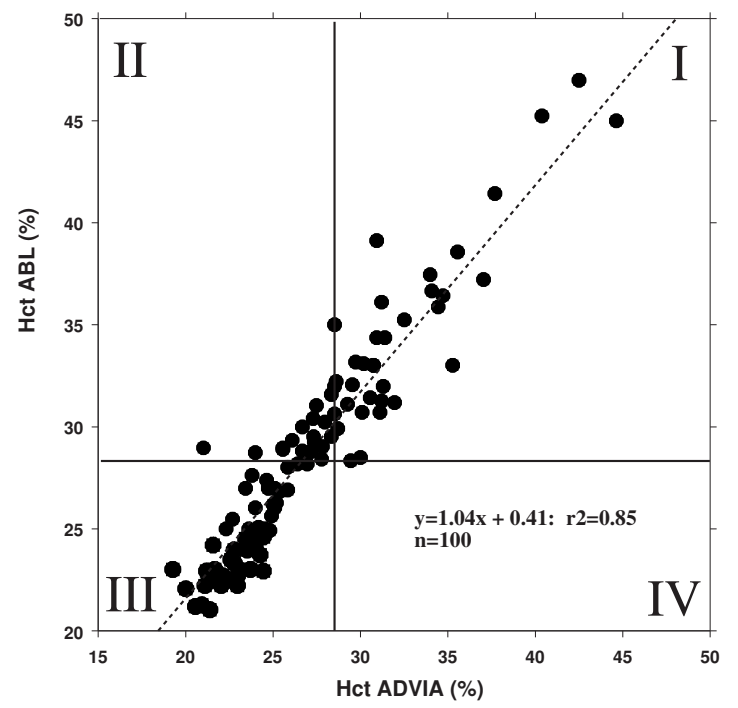

Figure 3 In 20 patients with predefined hematocrit target of $28 \%$ ( $n=100$ values) both methods correlated well with $r^{2}=0.8$, a kappa of 0.58 reflected a fair agreement of the methods. Values located within square III below $28 \%$ reflect patients who require RBC transfusions according to both methods of analysis, whereas square II shows 21 patients (21\%) in whom hematocrit determined by the central laboratory would have suggested RBC transfusion while blood gas analysis would not have indicated RBC transfusion. 
hospitals in multi-center trials [1]-[3]. In this context, it is of critical importance to use the same analytical procedure.

Analytical procedures are strongly dependent on in vivo and in vitro influences: In this context, the preanalytic phase is crucial for the subsequent analytic process[11] and most importantly for the interpretation of the obtained results. Point-of-care testing (POCT) has been introduced in clinical routine to provide analytical results more rapidly and to allow shorter therapeutic response intervals. For POCT systems like the ABLflex 800, preanalytic steps are decisive which include correct blood withdrawal with discarding of the first sample and immediate analysis to prevent hemodilution and stability of the specimens, respectively. Inappropriate handling and prolonged delay will result in sedimentation of RBC and will interfere with subsequent analysis. Details for preanalytics in POCT are described by Hicks et al [12]. Another important factor is the time span from blood withdrawal to their processing within the analyzer to optimize stability of hematological analysis which is time-dependent and hematology analyzer dependent[13]. In our ICU the nursing staff is trained in standardized blood withdrawal and immediate blood gas analysis within minutes after blood collection.

The samples for the ADVIA 2120 are directly sent to the hematology laboratory, marked "emergency" which guarantees immediate processing, providing results within one hour. The pre-analytic process and the rest of the testing in the laboratory is highly standardized. Thus, assuming that pre-analytic errors occurred, we would rather have to consider this a systematic error.

The ADVIA 2120 method may be influenced by hemolytic samples or lipemia leading to falsely elevated hematocrit levels, in contrast cold antibodies can decrease hematocrit. In addition, polyglobulia may affect the measurement [14]. In our population polyglobulia or lipemia was not present; cold antibodies were not searched for routinely.

The International Committee for Standardization in Hematology (ICSH) recommends the cyanmethemoglobin method as a reference method for hemoglobin measurement[7].

Although blood gas analyzers as POCT methods are increasingly used to provide rapid analysis of hematocrit in ICU, emergency departments, and operating theaters, data showing reliability between the different methods is scarce. A Belgian multicenter study compared different POCT methods to standard laboratory analyzer and found differences from 0.6 to $4.1 \%$ [15] which was corroborated by two other studies[16,17].

To date, we lack detailed studies addressing the impact of different analytical methods on the transfusion management. Although both tested methods showed a fair to good agreement, approximately $21 \%$ of our samples might lead to a different transfusion management. Taken together, apart from clinical parameters transfusion triggers must not only be defined as simple values but must be considered as method-dependent parameters. In the literature none of the landmark papers [1-3] mentioned the hemoglobin analyzing method. This, in turn, could in theory, lead to different results of these studies. Most importantly, multi-center trials must use the same method of analysis to avoid false transfusions. Concerning the ICSH guidelines which recommend the cyanmethemoglobin method as a standard method, the ADVIA 2120 method might be more appropriate to measure hemoglobin/hematocrit. Nevertheless, transfusing according to the ADVIA 2120 method would increase the transfusion rate in our patients with its additional risks.

\section{Competing interests}

The authors declare that they have no competing interests.

\section{Authors' contributions}

RS and MBo helped to design the study and drafted parts of the manuscript, LMA and RAS collected the data, TAN and SRC drafted parts of the manuscript, JFS and JF performed the statistics and MB designed the study, drafted parts of the manuscript and was the leader of the project.

All authors read and approved the final manuscript.

\section{Acknowledgements}

This study was supported in parts by the SUVA to JFS and RS.

\section{Author Details}

1Surgical Intensive Care Medicine, University Hospital of Zurich, CH 8091 Zurich, Switzerland and ${ }^{2}$ Department of Internal Medicine, Division of Hematology, University Hospital of Zurich, CH 8091 Zurich, Switzerland

Received: 20 November 2009 Accepted: 9 March 2010

Published: 9 March 2010

\section{References}

1. Hebert PC, Wells G, Blajchman MA, Marshall J, Martin C, Pagliarello G, Tweeddale M, Schweitzer I, Yetisir E: A multicenter, randomized, controlled clinical trial of transfusion requirements in critical care. Transfusion Requirements in Critical Care Investigators, Canadian Critical Care Trials Group. N Engl J Med 1999, 340(6):409-417.

2. Lacroix J, Hebert PC, Hutchison JS, Hume HA, Tucci M, Ducruet T, Gauvin F, Collet JP, Toledano BJ, Robillard P, et al:: Transfusion strategies for patients in pediatric intensive care units. N Engl J Med 2007, 356(16):1609-1619.

3. Kirpalani H, Whyte RK, Andersen C, Asztalos EV, Heddle N, Blajchman MA, Peliowski A, Rios A, LaCorte M, Connelly R, et al:: The Premature Infants in Need of Transfusion (PINT) study: a randomized, controlled trial of a restrictive (low) versus liberal (high) transfusion threshold for extremely low birth weight infants. J Pediatr 2006, 149(3):301-307.

4. Felding P, Petersen PH, Horder M: The stability of blood, plasma and serum constituents during simulated transport. Scandinavian journal of clinical and laboratory investigation 1981, 41(1):35-40.

5. Felding P, Tryding N, Hyltoft Petersen P, Horder M: Effects of posture on concentrations of blood constituents in healthy adults: practical application of blood specimen collection procedures recommended by the Scandinavian Committee on Reference Values. Scandinavian journal of clinical and laboratory investigation 1980, 40(7):615-621.

6. Malin MJ, Sclafani LD, Wyatt JL: Evaluation of 24-second cyanidecontaining and cyanide-free methods for whole blood hemoglobin on the Technicon $\mathrm{H}^{*} 1 \mathrm{TM}$ analyzer with normal and abnormal blood samples. American journal of clinical pathology 1989, 92(3):286-294. 
7. Zwart A, van Assendelft OW, Bull BS, England JM, Lewis SM, Zijlstra WG: Recommendations for reference method for haemoglobinometry in human blood (ICSH standard 1995) and specifications for international haemiglobinocyanide standard (4th edition). Journal of clinical pathology 1996, 49(4):271-274.

8. Bland JM, Altman DG: Agreement between methods of measurement with multiple observations per individual. Journal of biopharmaceutical statistics 2007, 17(4):571-582.

9. Myles PS, Cui J: Using the Bland-Altman method to measure agreement with repeated measures. British journal of anaesthesia 2007, 99(3):309-311

10. Cohen J: A coefficient of agreement for nominal scales. Educational and Psychological Measurement 1960:37-46.

11. Narayanan S: The preanalytic phase. An important component of laboratory medicine. American journal of clinical pathology 2000, 113(3):429-452.

12. Hicks JM, Haeckel R, Price CP, Lewandrowski K, Wu AH: Recommendations and opinions for the use of point-of-care testing for hospitals and primary care: summary of a 1999 symposium. Clinica chimica acta; international journal of clinical chemistry 2001, 303(1-2):1-17.

13. Imeri F, Herklotz R, Risch L, Arbetsleitner C, Zerlauth M, Risch GM, Huber AR: Stability of hematological analytes depends on the hematology analyser used: a stability study with Bayer Advia 120, Beckman Coulter LH 750 and Sysmex XE 2100. Clinica chimica acta; international journal of clinical chemistry 2008, 397(1-2):68-71.

14. Steen G, Vermeer HJ, Naus AJ, Goevaerts B, Agricola PT, Schoenmakers $\mathrm{CH}$ : Multicenter evaluation of the interference of hemoglobin, bilirubin and lipids on Synchron LX-20 assays. Clin Chem Lab Med 2006, 44(4):413-419.

15. Harris N, Jou JM, Devoto G, Lotz J, Pappas J, Wranovics D, Wilkinson M, Fletcher SR, Kratz A: Performance evaluation of the ADVIA 2120 hematology analyzer: an international multicenter clinical trial. $\mathrm{Lab}$ Hematol 2005, 11(1):62-70.

16. Steinfelder-Visscher J, Weerwind PW, Teerenstra S, Brouwer MH: Reliability of point-of-care hematocrit, blood gas, electrolyte, lactate and glucose measurement during cardiopulmonary bypass. Perfusion 2006, 21(1):33-37.

17. Walton $\mathrm{HG}$, Boucher DM, Marroquin R: Comparison of blood gas and electrolyte test results from the Gem-Premier and the ABL-70 versus a conventional laboratory analyzer. The Journal of extra-corporeal technology 2003, 35(1):24-27.

doi: 10.1186/1756-0500-3-65

Cite this article as: Bosshart et al., Two different hematocrit detection methods: Different methods, different results? BMC Research Notes 2010, 3:65

Submit your next manuscript to BioMed Centra and take full advantage of:

- Convenient online submission

- Thorough peer review

- No space constraints or color figure charges

- Immediate publication on acceptance

- Inclusion in PubMed, CAS, Scopus and Google Scholar

- Research which is freely available for redistribution

Submit your manuscript at www.biomedcentral.com/submit
C Biomed Central 